\title{
Faults Estimation and Decentralized Control for a Networked Control System with Specified Faulty Actuators
}

\author{
Ching-Min Lee
}

\begin{abstract}
In this paper, we investigate a problem related to an estimation and decentralized control for faults for a networked control system whose configuration between subsystems is with variable structures. Taking advantage of the variable structures, the NCS could be formulated as a distributed type or a decentralized one, or as a time-varying kind switching between these two types. In the NCS, subsystems subject to network induced phenomena, such as networked induced time-varying delay, packets losses, and some faults caused by faulty actuators. To deal with these considerations, we introduce a fault dynamics and derive a set of LMI-based delay-dependent conditions for a stability analysis problem to the corresponding augmented estimation system for faults. Based on the resultant stability conditions, we also derive a set of LMI-based synthesis conditions for designing estimators and decentralized controllers to cope with faults. At the end of this paper, a numerical example is presented to show the feasibility and efficiency for the proposed method.
\end{abstract}

Index Terms-Decentralized control, faults estimation, LMI, NCS.

\section{INTRODUCTION}

Networked control systems (NCSs) are those controlled systems whose components or devices connected with (wireless and/or wire) transmission networks. Comparing with traditional control systems, NCS has many advantages in cost-effectiveness, power requirements, simple installation and maintenance and so on. With the rapid and wide applications, such as Internet of Things [1], topics related to NCSs have been gotten growing attention, not only in theoretical developments but also in practical engineering and living around [2], [3]. Except for a single or a small-scale $\mathrm{NCS}$, with the growing scale of an NCS, according to the number of controller or whether controllers belonging to different subsystems of NCS exchange information to each other, the types of an NCS consisting of two or more subsystems can be grouped into centralized, decentralized, and distributed ones [4]. Each type of an NCS has their own advantages and disadvantages for usage, as well as challenges for development. Besides, different from traditional control systems, NCSs have many unavoidable situations including networked induced delay, packets losses, disorder packets etc., which leads to challenges when ones

Manuscript received April 26, 2019; revised November 11, 2019. This work is supported by Ministry of Science and Technology of Taiwan under Grant MOST 106-2221-E-214-009 and 107-2221-E-214-031.

Ching-Min Lee is with the Department of Electrical Engineering, I-Shou University, Kaohsiung City, Taiwan (e-mail: cmlee@isu.edu.tw). consider related topics of NCSs. Therefore, not only filtering problems [5], stability analysis problems [6], controller design problems [7] for an NCS are investigated continuously, but also more and more various requirements related to NCSs are focused, especially for the requirements about the quality of service, security, reliability and robustness under various environments, limitations and conditions [8]-[10]. Fortunately, for the most of these requirements, their essential problems are related to the fault detection/diagnosis, estimation, and fault tolerant control problems for NCSs [11], [12]. Consequentially, in this paper, we would like to simultaneously consider a faults estimation and control problem for an NCS with specified faulty actuators. In order to be capable to consider a more general NCS, we introduce an integrated NCS form with variable structures which could be used to consider a decentralized NCS, or a distributed one, or a time-varying kind switching between these two types.

The preliminary results of those herein were presented in the conference paper [13], which focused on stability and performance for the cases with interacting and non-interacting states between different subsystems, respectively. The main extensions of this paper include a more general case that both for states and for faults signals interacting and non-interacting are considered; the details of the proof for the resultant conditions for the stability analysis problem are provided, and a modified numerical example is given.

Notation: Matrices have compatible sizes for algebraic operations if their sizes are not explicitly stated in this paper. The inequality $\mathbf{M}>(<) \mathbf{0}$ means that the matrix $\mathbf{M}$ is symmetric and positive (negative) definite. For a square matrix $\mathbf{N}, \operatorname{sym}(\mathbf{N})=\mathbf{N}+\mathbf{N}^{\mathrm{T}}$, where ${ }^{\mathrm{T}}$ is the transpose of a matrix. The diag $\{$. $\}$ represents a block diagonal matrix. I and $\mathbf{0}$ are identity and zero matrices, respectively, with appropriate size. $l_{2}[0, \infty)$ is the space of square-summable vectors. In a symmetric matrix, * represents the transpose of an off-diagonal submatrix. $\mathrm{E}\{\bullet\}$ is an expected value.

\section{PROBLEM Formulation AND PRELIMINARIES}

\section{A. Problem Formulation}

Consider a networked control system, shown in Fig. 1, consisting of $s$ distributed subsystems around a communication network. Each subsystem has its own local components including a plant, some actuators, controllers, and sensors. The configuration between subsystems is 
variable structure, which means that sometimes states of one subsystem could exchange their information to the other subsystems, sometimes they only have their own data. Similar cases also appear for the faults induced by actuators of each subsystem. In addition, controllers for each subsystem are located around the network but to be local for its own plant. The dynamics of the integrated NCS therefore can be described as

$$
\boldsymbol{\Sigma}_{p}:\left\{\begin{aligned}
\mathbf{x}(k+1) & =\mathbf{A}^{\left(\rho_{k}\right)} \mathbf{x}(k)+\mathbf{B u}(k)+\mathbf{F}^{\left(\rho_{k}\right)} \mathbf{f}(k)+\mathbf{D} \boldsymbol{\omega}(k) \\
\mathbf{y}(k) & =\mathbf{C}^{\left(\rho_{k}\right)} \mathbf{x}(k),
\end{aligned}\right.
$$

where $\quad \mathbf{x}(k)=\left[\mathbf{x}_{1}^{\mathrm{T}}(k) \mathbf{x}_{2}^{\mathrm{T}}(k) \cdots \mathbf{x}_{s}^{\mathrm{T}}(k)\right]^{\mathrm{T}} \in \mathfrak{R}^{n_{s}} \quad$ is the state vector; $\mathbf{u}(k)=\left[\mathbf{u}_{1}^{\mathrm{T}}(k) \mathbf{u}_{2}^{\mathrm{T}}(k) \cdots \mathbf{u}_{s}^{\mathrm{T}}(k)\right]^{\mathrm{T}} \in \mathfrak{R}^{p_{s}}$ is the input vector; $\mathbf{y}(k)=\left[\mathbf{y}_{1}^{\mathrm{T}}(k) \mathbf{y}_{2}^{\mathrm{T}}(k) \cdots \mathbf{y}_{s}^{\mathrm{T}}(k)\right]^{\mathrm{T}} \in \mathfrak{R}^{q_{s}} \quad$ is the measurement output vector at plants; $\boldsymbol{\omega}(k) \in \mathfrak{R}^{w_{s}} \subset l_{2}[0, \infty)$ is the disturbance vector, and $\mathbf{f}(k)=\left[\mathbf{f}_{1}^{\mathrm{T}}(k) \mathbf{f}_{2}^{\mathrm{T}}(k) \cdots \mathbf{f}_{s}^{\mathrm{T}}(k)\right]^{\mathrm{T}} \in \mathfrak{R}^{m_{s}}$ denotes the faults induced by faulty actuators. Moreover, for the $i^{\text {th }} \quad$ subsystem, $\quad \mathbf{x}_{i}(k) \in \mathfrak{R}^{\bar{n}_{i}}, \mathbf{u}_{i}(k) \in \mathfrak{R}^{\bar{p}_{i}}, \mathbf{f}_{i}(k) \in \mathfrak{R}^{\bar{m}_{i}}$, $\mathbf{y}_{i}(k) \in \mathfrak{R}^{\bar{q}_{i}}, i \in S=\{1, \ldots, s\}$. System matrices in (1) are

$$
\begin{aligned}
\mathbf{A}^{\left(\rho_{k}\right)} & =\left[\begin{array}{ccc}
\mathbf{A}_{1} & \cdots & \rho_{1 s}^{k} \mathbf{A}_{1 s} \\
\vdots & \ddots & \vdots \\
\rho_{s 1}^{k} \mathbf{A}_{s 1} & \cdots & \mathbf{A}_{s}
\end{array}\right], \mathbf{F}^{\left(\rho_{k}\right)}=\left[\begin{array}{ccc}
\mathbf{F}_{1} & \cdots & \rho_{1 s}^{k} \mathbf{F}_{1 s} \\
\vdots & \ddots & \vdots \\
\rho_{s 1}^{k} \mathbf{F}_{s 1} & \cdots & \mathbf{F}_{s}
\end{array}\right], \\
\mathbf{C}^{\left(\rho_{k}\right)} & =\left[\begin{array}{ccc}
\mathbf{C}_{1} & \cdots & \rho_{1 s}^{k} \mathbf{C}_{1 s} \\
\vdots & \ddots & \vdots \\
\rho_{s 1}^{k} \mathbf{C}_{s 1} & \cdots & \mathbf{C}_{s}
\end{array}\right], \begin{array}{l}
\mathbf{B}=\operatorname{diag}\left\{\mathbf{B}_{1}, \cdots, \mathbf{B}_{s}\right\}, \\
\mathbf{D}=\operatorname{diag}\left\{\mathbf{D}_{1}, \cdots, \mathbf{D}_{s}\right\} .
\end{array}
\end{aligned}
$$

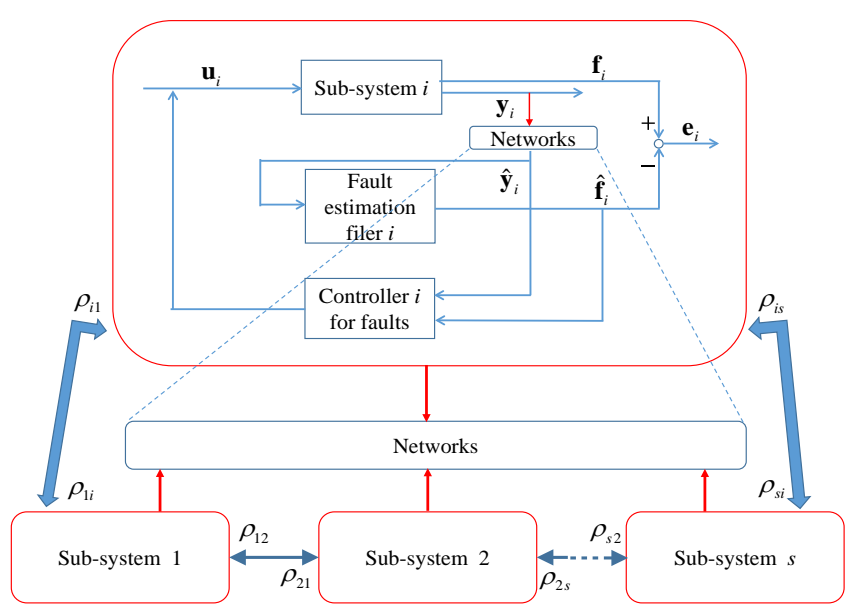

Fig. 1. Networked control system with variable structures.

The time-varying coupling parameter $\rho_{i j}^{k} \in \rho_{k}=\{0,1\}, i, j \in S$, denotes the coupling relation between subsystem $j$ and $i$, which also represents whether subsystem $j$ affects subsystem $i$. For all $k$, if $\rho_{i j}=0$ for all subsystems, the networked control system is decentralized, while it is distributed if there exist some $\rho_{i j}=1$. Moreover, the formulation here could involve these two types of configuration varying with $k$. In such cases, it is unavoidable that the NCS would be subject to networked induced time-varying delay, packets losses, as well as faults induced by some faulty actuators of subsystems. Due to prepared knowledge and well understanding for faults about actuators, the integrated faults dynamics can be further introduced as

$$
\Sigma_{F}:\left\{\begin{aligned}
\mathbf{x}_{f}(k+1) & =\mathbf{A}_{f} \mathbf{x}_{f}(k)+\mathbf{B}_{f} \overline{\mathbf{f}}(k), \\
\mathbf{f}(k) & =\mathbf{C}_{f} \mathbf{x}_{f}(k),
\end{aligned}\right.
$$

where $\quad \mathbf{x}_{f}(k)=\left[\mathbf{x}_{f 1}^{\mathrm{T}}(k) \cdots \mathbf{x}_{f s}^{\mathrm{T}}(k)\right]^{\mathrm{T}} \in \mathfrak{R}^{n_{f}} \quad$ is the states; $\overline{\mathbf{f}}(k)=\left[\overline{\mathbf{f}}_{1}^{\mathrm{T}}(k) \cdots \overline{\mathbf{f}}_{s}^{\mathrm{T}}(k)\right]^{\mathrm{T}} \in \mathfrak{R}^{l_{f}}$ is the sources of faults, and matrices $\quad \mathbf{A}_{f}=\operatorname{diag}\left\{\mathbf{A}_{f 1}, \cdots, \mathbf{A}_{f s}\right\}, \quad \mathbf{B}_{f}=\operatorname{diag}\left\{\mathbf{B}_{f 1}, \cdots, \mathbf{B}_{f s}\right\}$, $\mathbf{C}_{f}=\operatorname{diag}\left\{\mathbf{C}_{f 1}, \cdots, \mathbf{C}_{f s}\right\}$. For each subsystem, its output signals are transmitted to remote devices via communication networks. Therefore, the remote output signals of subsystem $i$ can be formulated as

$$
\begin{aligned}
& \hat{\mathbf{y}}_{i}(k)=\alpha_{i}(k) \mathbf{y}_{i}\left(k-\tau_{i}(k)\right), \quad 0 \leq \tau_{m i} \leq \tau_{i}(k) \leq \tau_{M i}, \\
& \mathbf{x}_{i}\left(k_{0}\right)=\boldsymbol{\Phi}_{i}^{0}\left(k_{0}\right), k_{0}=-\tau_{M_{i}},-\tau_{M_{i}}+1, \cdots, 1,0 . \quad i \in S .
\end{aligned}
$$

where the time-varying delay $\tau_{i}(k) \in \tau(k)$, and $\tau_{m i}, \tau_{M i}$ are the lower and upper bounds of $\tau_{i}(k)$, respectively. $\boldsymbol{\Phi}_{i}^{0}\left(k_{0}\right)$ is the initial state vector on $\left[-\tau_{M i}, 0\right]$. The time-varying parameter $\alpha_{i}(k)$ denotes the event of packet lost or received, which is an independent Bernoulli process. The expected value of $\alpha_{i}(k)$ is supposed to be

$$
\begin{aligned}
& \left\{\operatorname{Prob}\left\{\alpha_{i}(k)=1\right\}=\mathrm{E}\left\{\alpha_{i}(k)=1\right\}=\alpha_{i}, \quad\right. \text { packet received, } \\
& \left\{\operatorname{Prob}\left\{\alpha_{i}(k)=0\right\}=\mathrm{E}\left\{\alpha_{i}(k)=0\right\}=1-\alpha_{i}\right. \text {, packet lost. }
\end{aligned}
$$

and $\alpha_{i} \in(0,1], i \in S$. The corresponding integrated remote output signals for the NCS in (1) can be written as

$$
\begin{aligned}
& \hat{\mathbf{y}}(k)=\boldsymbol{\alpha}(k) \mathbf{y}(k-\tau(k)), \quad 0 \leq \tau_{m} \leq \tau(k) \leq \tau_{M}, \\
& \mathbf{x}(k)=\boldsymbol{\Phi}^{0}(k), k=-\tau_{M},-\tau_{M}+1, \cdots, 1,0 .
\end{aligned}
$$

where $\quad \boldsymbol{\alpha}(k)=\operatorname{diag}\left\{\alpha_{1}(k) \mathbf{I}_{\bar{q}_{1}}, \cdots, \alpha_{s}(k) \mathbf{I}_{\bar{q}_{s}}\right\}, \quad \tau_{m}=\min _{i \in S} \tau_{m i}$, $\tau_{M}=\max _{i \in S} \tau_{M i}$. For the NCS in (1), in order to estimate the appearing faults $\mathbf{f}(k)$, a desired integrated remote fault estimator is formulated as

$$
\Sigma_{F f}:\left\{\begin{aligned}
\hat{\mathbf{x}}(k+1) & =\hat{\mathbf{A}} \hat{\mathbf{x}}(k)+\hat{\mathbf{B}} \hat{\mathbf{y}}(k), \\
\hat{\mathbf{f}}(k) & =\hat{\mathbf{C}} \hat{\mathbf{x}}(k),
\end{aligned}\right.
$$

where $\hat{\mathbf{x}}(k)=\left[\hat{\mathbf{x}}_{1}^{\mathrm{T}}(k) \ldots \hat{\mathbf{x}}_{s}^{\mathrm{T}}(k)\right]^{\mathrm{T}} \in \mathfrak{R}^{n_{e}}\left(\hat{\mathbf{x}}_{i} \in \mathfrak{R}^{\bar{n}_{e i}}, i \in S\right)$ is the integrated estimator state vector; $\hat{\mathbf{f}}(k) \in \mathfrak{R}^{m_{s}}$ is the estimated faults; matrices $\hat{\mathbf{A}}=\operatorname{diag}\left\{\hat{\mathbf{A}}_{1}, \ldots, \hat{\mathbf{A}}_{s}\right\}, \quad \hat{\mathbf{B}}=\operatorname{diag}\left\{\hat{\mathbf{B}}_{1}, \cdots, \hat{\mathbf{B}}_{s}\right\}$, $\hat{\mathbf{C}}=\operatorname{diag}\left\{\hat{\mathbf{C}}_{1}, \cdots, \hat{\mathbf{C}}_{s}\right\}$ are the estimator gains to be designed. On the other hand, according to the estimated faults, in order to maintain the fundamental function, such as stability of the NCS suffering from these faults, a desired remote output-feedback controller is formulated as

$$
\begin{aligned}
\mathbf{u}(k) & =\mathbf{K} \hat{\mathbf{y}}(k)+\mathbf{K}_{f} \hat{\mathbf{f}}(k), \\
\mathbf{K} & =\operatorname{diag}\left\{\mathbf{K}_{1}, \ldots, \mathbf{K}_{s}\right\} \in \mathfrak{R}^{p_{s} \times q_{s}}, \quad \mathbf{K}_{i} \in \mathfrak{R}^{\bar{p}_{i} \times \bar{q}_{i}}, \\
\mathbf{K}_{f} & =\operatorname{diag}\left\{\mathbf{K}_{f 1}, \ldots, \mathbf{K}_{f s}\right\} \in \mathfrak{R}^{p_{s} \times m_{s}}, \mathbf{K}_{f i} \in \mathfrak{R}^{\bar{p}_{i} \times \bar{m}_{i}}, i \in S .
\end{aligned}
$$


Take into account the NCS in (1) together with the remote fault estimator in (6) and the output-feedback controller in (7), the augmented estimation dynamics for faults can be organized as

$$
\Sigma_{F E C}:\left\{\begin{aligned}
\overline{\mathbf{x}}(k+1) & =\overline{\mathbf{A}}^{\left(\rho_{k}\right)} \overline{\mathbf{x}}(k)+\underline{\boldsymbol{\alpha}}(k) \overline{\mathbf{B}}^{\left(\rho_{k}\right)} \overline{\mathbf{x}}(k-\tau(k))+\overline{\mathbf{H}} \overline{\boldsymbol{\omega}}(k), \\
\mathbf{e}(k) & =\overline{\mathbf{C}} \overline{\mathbf{x}}(k),
\end{aligned}\right.
$$

where $\mathbf{e}(k)=\mathbf{f}(k)-\hat{\mathbf{f}}(k)$ is the estimation errors of faults, and

$$
\begin{aligned}
& \overline{\mathbf{x}}(k)=\left[\begin{array}{c}
\mathbf{x}_{p}(k) \\
\hat{\mathbf{x}}(k)
\end{array}\right], \quad \mathbf{x}_{p}(k)=\left[\begin{array}{c}
\mathbf{x}(k) \\
\mathbf{x}_{f}(k)
\end{array}\right], \quad \overline{\boldsymbol{\omega}}(k)=\left[\begin{array}{c}
\boldsymbol{\omega}(k) \\
\overline{\mathbf{f}}(k)
\end{array}\right], \\
& \overline{\mathbf{A}}^{\left(\rho_{k}\right)}=\left[\begin{array}{cc}
\mathbf{A}_{p}^{\left(\rho_{k}\right)} & \mathbf{B} \mathbf{K}_{f} \hat{\mathbf{C}} \\
\mathbf{0} & \hat{\mathbf{A}}
\end{array}\right], \quad \overline{\mathbf{B}}^{\left(\rho_{k}\right)}=\left[\begin{array}{cc}
\mathbf{A}_{p d}^{\left(\rho_{k}\right)} & \mathbf{0} \\
\mathbf{B}_{p d}^{\left(\rho_{k}\right)} & \mathbf{0}
\end{array}\right], \quad \overline{\mathbf{H}}=\left[\begin{array}{c}
\mathbf{D}_{p} \\
\mathbf{0}
\end{array}\right], \\
& \overline{\mathbf{C}}=\left[\begin{array}{ll}
\mathbf{C}_{p} & -\hat{\mathbf{C}}
\end{array}\right], \quad \mathbf{C}_{p}=\left[\begin{array}{ll}
\mathbf{0} & \mathbf{C}_{f}
\end{array}\right], \quad \mathbf{B}_{p d}^{\left(\rho_{k}\right)}=\left[\begin{array}{ll}
\hat{\mathbf{B}} \mathbf{C}^{\left(\rho_{k}\right)} & \mathbf{0}
\end{array}\right], \\
& \mathbf{A}_{p}^{\left(\rho_{k}\right)}=\left[\begin{array}{cc}
\mathbf{A}^{\left(\rho_{k}\right)} & \mathbf{F}^{\left(\rho_{k}\right)} \mathbf{C}_{f} \\
\mathbf{0} & \mathbf{A}_{f}
\end{array}\right], \mathbf{A}_{p d}^{\left(\rho_{k}\right)}=\left[\begin{array}{cc}
\mathbf{B K} \mathbf{C}^{\left(\rho_{k}\right)} & \mathbf{0} \\
\mathbf{0} & \mathbf{0}
\end{array}\right], \mathbf{D}_{p}=\left[\begin{array}{cc}
\mathbf{D} & \mathbf{0} \\
\mathbf{0} & \mathbf{B}_{f}
\end{array}\right], \\
& \underline{\boldsymbol{\alpha}}(k)=\operatorname{diag}\left\{\overline{\boldsymbol{\alpha}}(k), \varepsilon \mathbf{I}_{n_{f}}, \hat{\boldsymbol{\alpha}}(k)\right\}, \quad \underline{\boldsymbol{\alpha}}=\operatorname{diag}\left\{\overline{\boldsymbol{\alpha}}, \varepsilon \mathbf{I}_{n_{f}}, \hat{\boldsymbol{\alpha}}\right\}, \varepsilon \neq 0,1 . \\
& \overline{\boldsymbol{\alpha}}(k)=\operatorname{diag}\left\{\alpha_{1}(k) \mathbf{I}_{\bar{n}_{1}}, \cdots, \alpha_{s}(k) \mathbf{I}_{\bar{n}_{s}}\right\}, \quad \overline{\boldsymbol{\alpha}}=\operatorname{diag}\left\{\alpha_{1} \mathbf{I}_{\bar{n}_{1}}, \cdots, \alpha_{s} \mathbf{I}_{\bar{n}_{s}}\right\}, \\
& \hat{\boldsymbol{\alpha}}(k)=\operatorname{diag}\left\{\alpha_{1}(k) \mathbf{I}_{\bar{n}_{e l}}, \cdots, \alpha_{s}(k) \mathbf{I}_{\bar{n}_{e s}}\right\}, \quad \hat{\boldsymbol{\alpha}}=\operatorname{diag}\left\{\alpha_{1} \mathbf{I}_{\bar{n}_{e 1}}, \cdots, \alpha_{s} \mathbf{I}_{\bar{n}_{e s}}\right\} \text {. }
\end{aligned}
$$

\section{B. Preliminaries and Objective}

In this subsection, we provide some definitions and useful lemmas for this paper.

Definition 1. The system in (8) is said to be stochastically stable if there exist real numbers $\{a, b\}>0$, such that

$$
\mathrm{E}\{\|\overline{\mathbf{x}}(k)\|\} \leq a,\left\|\overline{\mathbf{x}}\left(k_{0}\right)\right\| \leq b, \quad k \in \mathbb{Z}, k_{0} \in\left[-\tau_{M}, 0\right], \tau_{M}>0
$$

Definition 2. Under zero initial conditions, the $H_{\infty}$ performance index for system (8) is defined as

$$
\sum_{k=0}^{\infty} \mathrm{E}\left\{\mathbf{e}^{\mathrm{T}}(k) \mathbf{e}(k)\right\} \leq \gamma^{2} \sum_{k=0}^{\infty} \mathrm{E}\left\{\overline{\boldsymbol{\omega}}^{\mathrm{T}}(k) \overline{\boldsymbol{\omega}}(k)\right\}
$$

for a prescribed positive scalar $\gamma$ and all nonzero $\overline{\boldsymbol{\omega}}(k)$.

Lemma 1 below, obtained via similar procedures of the proof for Theorem 1 in [14], is useful for resulting in resultant LMI-based synthesis conditions.

Lemma 1. For a system matrix $\mathbf{A}_{0}$, the following two conditions are equivalent.

(a) There exists a positive definite matrix $\mathbf{M}$, such that

$$
f(\mathbf{M})>\mathbf{0}, \quad \mathbf{A}_{0}^{\mathrm{T}} \mathbf{M} \mathbf{A}_{0}-f(\mathbf{M})<\mathbf{0} .
$$

(b) There exists a positive definite matrix $\mathbf{M}$ and a nonsingular matrix $\boldsymbol{\Sigma}$, such that

$$
\left[\begin{array}{cc}
-f(\mathbf{M}) & \mathbf{A}_{0}^{\mathrm{T}} \boldsymbol{\Sigma} \\
\boldsymbol{\Sigma}^{\mathrm{T}} \mathbf{A}_{0} & \mathbf{M}-\boldsymbol{\Sigma}-\boldsymbol{\Sigma}^{\mathrm{T}}
\end{array}\right]<\mathbf{0} .
$$

In addition, there are some assumptions for the NCS in this paper.

Assumption 1. The communication network only exists in sensors-to-controllers/estimators.
Assumption 2. The matrix B in (1) is full column rank.

The objective of this paper is to design a set feasible fault estimators and controllers for each subsystem constructing the integrated networked control system, such that the corresponding estimation error dynamics for faults is stochastically stable, and satisfies a desired $H_{\infty}$ performance index under zero initial conditions.

In order to deal with the fault estimation and control problems for the NCS in (1), recall the parameter $\rho_{i j}^{k} \in \rho_{k}=\{0,1\}$ in (2) and (9). The time-varying matrices $\left\{\mathbf{A}^{\left(\rho_{k}\right)}, \mathbf{F}^{\left(\rho_{k}\right)}, \mathbf{C}^{\left(\rho_{k}\right)}, \overline{\mathbf{A}}^{\left(\rho_{k}\right)}, \overline{\mathbf{B}}^{\left(\rho_{k}\right)}\right\}$ can be considered as belonging to a convex compact set of polytopic type, that is,

$$
\begin{aligned}
& \psi^{\rho_{k}}=\left(\mathbf{A}^{\left(\rho_{k}\right)}, \mathbf{F}^{\left(\rho_{k}\right)}, \mathbf{C}^{\left(\rho_{k}\right)}, \mathbf{A}_{p}^{\left(\rho_{k}\right)}, \mathbf{A}_{p d}^{\left(\rho_{k}\right)}, \mathbf{B}_{p d}^{\left(\rho_{k}\right)}, \overline{\mathbf{A}}^{\left(\rho_{k}\right)}, \overline{\mathbf{B}}^{\left(\rho_{k}\right)}\right) \in \varphi_{\rho_{k}}, \\
& \varphi_{\rho_{k}}=\left\{\psi^{\rho_{k}} \mid \psi^{\rho_{k}}=\sum_{l=1}^{v} \beta_{l} \psi_{\rho_{k}}^{l}, \sum_{l=1}^{v} \beta_{l}=1, \beta_{l} \geq 0\right\},
\end{aligned}
$$

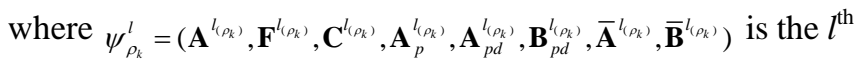
vertex of the polyhedral domain $\varphi_{\rho_{k}}$ and $l \in \Upsilon=\{1,2, \ldots, v\}$. For simplicity, we use $\psi^{l}=\left(\mathbf{A}^{l}, \mathbf{F}^{l}, \mathbf{C}^{l}, \mathbf{A}_{p}^{l}, \mathbf{A}_{p d}^{l}, \mathbf{B}_{p d}^{l}, \overline{\mathbf{A}}^{l}, \overline{\mathbf{B}}^{l}\right)$ to denote $\psi^{\rho_{k}}$ in the following sections.

\section{MAIN RESULTS}

In this section, a stability analysis problem for the augmented estimation dynamics in (8) is considered, and the resultant conditions are derived in Theorem 1 below. Based on the stability conditions in Theorem 1 , the design problem of the estimator and controller for faults is then developed, and the resultant conditions under linear matrix inequality framework are presented in Theorem 2.

\section{A. Conditions for a Stability Analysis Problem}

Theorem 1. Suppose $\left\{\hat{\mathbf{A}}, \hat{\mathbf{B}}, \hat{\mathbf{C}}, \mathbf{K}, \mathbf{K}_{f}\right\}$ are given. If there exist feasible matrices $\boldsymbol{\Gamma}_{i}, \boldsymbol{\Psi}_{i}(i=1,2,3,4)$; positive definite matrices $\mathbf{Q}, \boldsymbol{\Phi}_{1}, \boldsymbol{\Phi}_{2}$ and positive definite diagonal matrices $\mathbf{P}, \boldsymbol{\Lambda}_{1}, \boldsymbol{\Lambda}_{2}$ satisfying the following LMIs (12) for all $l \in \Upsilon$, then the system $\Sigma_{F E C}$ in (8) is stochastically stable and, under zero initial conditions, satisfied the $H_{\infty}$ performance index defined in (10)

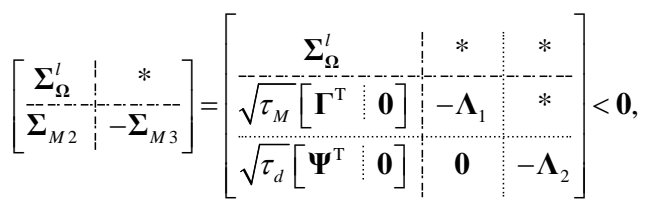

where

$$
\begin{aligned}
& \boldsymbol{\Gamma}^{\mathrm{T}}=\left[\begin{array}{llll}
\boldsymbol{\Gamma}_{1}^{\mathrm{T}} & \boldsymbol{\Gamma}_{2}^{\mathrm{T}} & \boldsymbol{\Gamma}_{3}^{\mathrm{T}} & \boldsymbol{\Gamma}_{4}^{\mathrm{T}}
\end{array}\right], \boldsymbol{\Psi}^{\mathrm{T}}=\left[\begin{array}{llll}
\boldsymbol{\Psi}_{1}^{\mathrm{T}} & \boldsymbol{\Psi}_{2}^{\mathrm{T}} & \boldsymbol{\Psi}_{3}^{\mathrm{T}} & \boldsymbol{\Psi}_{4}^{\mathrm{T}}
\end{array}\right], l \in \Upsilon, \\
& \boldsymbol{\Sigma}_{\mathbf{\Omega}}^{l}=\left[\begin{array}{ccccc}
\boldsymbol{\Sigma}_{\mathbf{\Omega} 11}^{l} & * & * & * & * \\
\boldsymbol{\Sigma}_{\mathbf{\Omega} 11} & \boldsymbol{\Sigma}_{\mathbf{\Omega} 22} & * & * & * \\
\boldsymbol{\Sigma}_{\mathbf{\Omega} 31}^{l} & \boldsymbol{\Sigma}_{\mathbf{\Omega 3 2}} & \boldsymbol{\Sigma}_{\mathbf{\Omega 3 3}}^{l} & * & * \\
\boldsymbol{\Sigma}_{\mathbf{\Omega} 41} & \boldsymbol{\Sigma}_{\mathbf{\Omega} 42} & \boldsymbol{\Sigma}_{\mathbf{\Omega} 43} & \boldsymbol{\Sigma}_{\mathbf{\Omega} 44} & * \\
\boldsymbol{\Sigma}_{\mathbf{\Omega} 51}^{l} & \mathbf{0} & \boldsymbol{\Sigma}_{\mathbf{\Omega} 53}^{l} & \mathbf{0} & \boldsymbol{\Sigma}_{\mathbf{\Omega} 55}^{l}
\end{array}\right] \text {, }
\end{aligned}
$$




$$
\begin{aligned}
& \boldsymbol{\Sigma}_{\mathbf{\Omega} 11}^{l}=\overline{\mathbf{A}}^{\mathrm{T} l} \mathbf{P} \overline{\mathbf{A}}^{l}-\mathbf{P}+\left(\tau_{d}+1\right) \mathbf{Q}+\overline{\mathbf{C}}^{\mathrm{T}} \overline{\mathbf{C}}+\tau_{M}\left(\overline{\mathbf{A}}^{l}-\mathbf{I}\right)^{\mathrm{T}} \boldsymbol{\Lambda}_{1}\left(\overline{\mathbf{A}}^{l}-\mathbf{I}\right) \\
& +\boldsymbol{\Phi}_{1}+\boldsymbol{\Phi}_{2}+\tau_{d}\left(\overline{\mathbf{A}}^{l}-\mathbf{I}\right)^{\mathrm{T}} \boldsymbol{\Lambda}_{2}\left(\overline{\mathbf{A}}^{l}-\mathbf{I}\right)+\operatorname{sym}\left(\boldsymbol{\Gamma}_{1}\right) ; \\
& \boldsymbol{\Sigma}_{\boldsymbol{\Omega} 21}=\boldsymbol{\Gamma}_{2}+\boldsymbol{\Psi}_{1}^{\mathrm{T}} ; \quad \boldsymbol{\Sigma}_{\mathbf{\Omega} 22}=\operatorname{sym}\left(\boldsymbol{\Psi}_{2}\right)-\boldsymbol{\Phi}_{2} ; \\
& \boldsymbol{\Sigma}_{\mathbf{\Omega} 31}^{l}=\overline{\mathbf{B}}^{\mathrm{T} l} \underline{\boldsymbol{\alpha}} \mathbf{P} \overline{\mathbf{A}}^{l}+\tau_{M} \overline{\mathbf{B}}^{\mathrm{T} l} \underline{\boldsymbol{\alpha}} \boldsymbol{\Lambda}_{1}\left(\overline{\mathbf{A}}^{l}-\mathbf{I}\right)+\tau_{d} \overline{\mathbf{B}}^{\mathrm{T} l} \underline{\boldsymbol{\alpha}}_{2}\left(\overline{\mathbf{A}}^{l}-\mathbf{I}\right)+\boldsymbol{\Gamma}_{3} ; \\
& \boldsymbol{\Sigma}_{\boldsymbol{\Omega} 33}^{l}=\overline{\mathbf{B}}^{\mathrm{Tl}} \underline{\boldsymbol{\alpha}} \overline{\mathbf{B}}^{l}+\tau_{M} \overline{\mathbf{B}}^{\mathrm{Tl}} \underline{\boldsymbol{\alpha}} \boldsymbol{\Lambda}_{1} \overline{\mathbf{B}}^{l}+\tau_{d} \overline{\mathbf{B}}^{\mathrm{Tl}} \underline{\boldsymbol{\alpha}} \boldsymbol{\Lambda}_{2} \overline{\mathbf{B}}^{l}-\mathbf{Q} ; \quad \boldsymbol{\Sigma}_{\boldsymbol{\Omega} 32}=\boldsymbol{\Psi}_{3} ; \\
& \boldsymbol{\Sigma}_{\boldsymbol{\Omega} 41}=\boldsymbol{\Gamma}_{4}-\boldsymbol{\Gamma}_{1}^{\mathrm{T}}-\boldsymbol{\Psi}_{1}^{\mathrm{T}} ; \boldsymbol{\Sigma}_{\mathbf{\Omega} 42}=\boldsymbol{\Psi}_{4}-\boldsymbol{\Gamma}_{2}^{\mathrm{T}}-\boldsymbol{\Psi}_{2}^{\mathrm{T}} \text {; } \\
& \boldsymbol{\Sigma}_{\mathbf{\Omega} 43}=-\boldsymbol{\Gamma}_{3}^{\mathrm{T}}-\boldsymbol{\Psi}_{3}^{\mathrm{T}} ; \quad \boldsymbol{\Sigma}_{\mathbf{\Omega} 44}=-\boldsymbol{\Phi}_{1}-\operatorname{sym}\left(\boldsymbol{\Gamma}_{4}\right)-\operatorname{sym}\left(\boldsymbol{\Psi}_{4}\right) ; \\
& \boldsymbol{\Sigma}_{\mathbf{\Omega} 51}^{l}=\overline{\mathbf{H}}^{\mathrm{T}} \mathbf{P} \overline{\mathbf{A}}^{l}+\tau_{M} \overline{\mathbf{H}}^{\mathrm{T}} \boldsymbol{\Lambda}_{1}\left(\overline{\mathbf{A}}^{l}-\mathbf{I}\right)+\tau_{d} \overline{\mathbf{H}}^{\mathrm{T}} \boldsymbol{\Lambda}_{2}\left(\overline{\mathbf{A}}^{l}-\mathbf{I}\right) ; \\
& \boldsymbol{\Sigma}_{\mathbf{\Omega} 33}^{l}=\overline{\mathbf{H}}^{\mathrm{T}} \mathbf{P} \underline{\boldsymbol{\alpha}} \overline{\mathbf{B}}^{l}+\tau_{M} \overline{\mathbf{H}}^{\mathrm{T}} \boldsymbol{\Lambda}_{1} \underline{\boldsymbol{\alpha}} \overline{\mathbf{B}}^{l}+\tau_{d} \overline{\mathbf{H}}^{\mathrm{T}} \boldsymbol{\Lambda}_{2} \underline{\boldsymbol{\alpha}} \overline{\mathbf{B}}^{l} ; \\
& \Sigma_{\mathbf{\Omega} 55}^{l}=\overline{\mathbf{H}}^{\mathrm{T}} \mathbf{P} \overline{\mathbf{H}}-\gamma^{2} \mathbf{I}+\tau_{M} \overline{\mathbf{H}}^{\mathrm{T}} \boldsymbol{\Lambda}_{1} \overline{\mathbf{H}}+\tau_{d} \overline{\mathbf{H}}^{\mathrm{T}} \boldsymbol{\Lambda}_{2} \overline{\mathbf{H}} ; \quad \tau_{d}=\tau_{M}-\tau_{m} .
\end{aligned}
$$

Proof. Consider a Lyapunov function candidate as

$$
V(k)=\sum_{i=1}^{6} V_{i}(k),
$$

for the augmented dynamics in (8), where

$$
\begin{aligned}
& V_{1}(k)=\overline{\mathbf{x}}^{\mathrm{T}}(k) \mathbf{P} \overline{\mathbf{x}}(k), \\
& V_{2}(k)=\sum_{i=k-\tau(k)}^{k-1} \overline{\mathbf{x}}^{\mathrm{T}}(i) \mathbf{Q} \overline{\mathbf{x}}(i)+\sum_{j=-\tau_{M}+1}^{-\tau_{m}} \sum_{i=k+j}^{k-1} \overline{\mathbf{x}}^{\mathrm{T}}(i) \mathbf{Q} \overline{\mathbf{x}}(i), \\
& V_{3}(k)=\sum_{i=k-\tau_{M}}^{k-1} \overline{\mathbf{x}}^{\mathrm{T}}(i) \mathbf{\Phi}_{1} \overline{\mathbf{x}}(i), \\
& V_{4}(k)=\sum_{i=k-\tau_{m}}^{k-1} \overline{\mathbf{x}}^{\mathrm{T}}(i) \boldsymbol{\Phi}_{2} \overline{\mathbf{x}}(i), \\
& V_{5}(k)=\sum_{j=-\tau_{M}}^{-1} \sum_{i=k+j}^{k-1} \overline{\mathbf{x}}_{e}^{\mathrm{T}}(i) \boldsymbol{\Lambda}_{1} \overline{\mathbf{x}}_{e}(i), \\
& V_{6}(k)=\sum_{j=-\tau_{M}}^{-\tau_{m}-1} \sum_{i=k+j}^{k-1} \overline{\mathbf{x}}_{e}^{\mathrm{T}}(i) \boldsymbol{\Lambda}_{2} \overline{\mathbf{x}}_{e}(i), \\
& \overline{\mathbf{x}}_{e}(i)=\overline{\mathbf{x}}(i+1)-\overline{\mathbf{x}}(i),
\end{aligned}
$$

with $\left\{\mathbf{P}, \mathbf{Q}, \boldsymbol{\Phi}_{1}, \mathbf{\Phi}_{2}, \boldsymbol{\Lambda}_{1}, \boldsymbol{\Lambda}_{2}\right\}>\mathbf{0}$, and $\mathbf{P}, \boldsymbol{\Lambda}_{1}, \boldsymbol{\Lambda}_{2}$ are diagonal. Denote $\quad \mathbf{X}(k)=\left[\begin{array}{lllll}\overline{\mathbf{x}}(k) & \overline{\mathbf{x}}(k-1) & \cdots & \overline{\mathbf{x}}\left(-\tau_{M}\right)\end{array}\right] . \quad$ For the independent random Bernoulli process $\alpha_{i}(k)$, we have $\mathrm{E}\left\{\left(\alpha_{i}(k)-\alpha_{i}\right)^{2}\right\}=\alpha_{i}\left(1-\alpha_{i}\right)$, and

$$
\begin{aligned}
& \mathrm{E}\left\{V_{1}(\overline{\mathbf{x}}(k+1)) \mid \mathbf{X}(k)\right\}-V_{1}(\overline{\mathbf{x}}(k)) \\
& =\overline{\mathbf{x}}^{\mathrm{T}}(k-\tau(k)) \overline{\mathbf{B}}^{\mathrm{Tl}}\left(\sqrt{\underline{\mathbf{a}}^{\mathrm{T}}} \mathbf{P} \sqrt{\underline{\boldsymbol{\alpha}}}-\underline{\mathbf{\alpha}}^{\mathrm{T}} \mathbf{P} \underline{\boldsymbol{\alpha}}\right) \overline{\mathbf{B}}^{\overline{\mathbf{x}}}(k-\tau(k)) \\
& -\overline{\mathbf{x}}^{\mathrm{T}}(k) \mathbf{P} \overline{\mathbf{x}}(k)+\left(\overline{\mathbf{A}} \overline{\mathbf{x}}(k)+\underline{\boldsymbol{\alpha}} \overline{\mathbf{B}}^{l} \overline{\mathbf{x}}(k-\tau(k))+\overline{\mathbf{H}} \overline{\boldsymbol{\omega}}(k)\right)^{\mathrm{T}} \mathbf{P} \\
& \left(\overline{\mathbf{A}}^{\prime} \overline{\mathbf{x}}(k)+\underline{\underline{\alpha}} \overline{\mathbf{B}}^{\prime} \overline{\mathbf{x}}(k-\tau(k))+\overline{\mathbf{H}} \overline{\mathbf{\omega}}(k)\right), \\
& \mathrm{E}\left\{V_{2}(\overline{\mathbf{x}}(k+1)) \mid \mathbf{X}(k)\right\}-V_{2}(\overline{\mathbf{x}}(k)) \\
& \leq\left(\tau_{d}+1\right) \overline{\mathbf{x}}^{\mathrm{T}}(k) \mathbf{Q} \overline{\mathbf{x}}(k)-\overline{\mathbf{x}}^{\mathrm{T}}(k-\tau(k)) \mathbf{Q} \overline{\mathbf{x}}(k-\tau(k)), \\
& \mathrm{E}\left\{V_{3}(\overline{\mathbf{x}}(k+1)) \mid \mathbf{X}(k)\right\}-V_{3}(\overline{\mathbf{x}}(k)) \\
& =\overline{\mathbf{x}}^{\mathrm{T}}(k) \boldsymbol{\Phi}_{1} \overline{\mathbf{x}}(k)-\overline{\mathbf{x}}^{\mathrm{T}}\left(k-\tau_{M}\right) \boldsymbol{\Phi}_{1} \overline{\mathbf{x}}\left(k-\tau_{M}\right), \\
& \mathrm{E}\left\{V_{4}(\overline{\mathbf{x}}(k+1)) \mid \mathbf{X}(k)\right\}-V_{4}(\overline{\mathbf{x}}(k)) \\
& =\overline{\mathbf{x}}^{\mathrm{T}}(k) \boldsymbol{\Phi}_{2} \overline{\mathbf{x}}(k)-\overline{\mathbf{x}}^{\mathrm{T}}\left(k-\tau_{m}\right) \boldsymbol{\Phi}_{2} \overline{\mathbf{x}}\left(k-\tau_{m}\right), \\
& \mathrm{E}\left\{V_{5}(\overline{\mathbf{x}}(k+1)) \mid \mathbf{X}(k)\right\}-V_{5}(k) \\
& =\tau_{M} \mathrm{E}\left\{\overline{\mathbf{x}}_{e}^{\mathrm{T}}(k) \boldsymbol{\Lambda}_{1} \overline{\mathbf{x}}_{e}(k)\right\}-\sum_{i=k-\tau_{M}}^{k-1} \overline{\mathbf{x}}_{e}^{\mathrm{T}}(i) \boldsymbol{\Lambda}_{1} \overline{\mathbf{x}}_{e}(i), \\
& \mathrm{E}\left\{V_{6}(\overline{\mathbf{x}}(k+1)) \mid \mathbf{X}(k)\right\}-V_{6}(k) \\
& =\tau_{d} \mathbb{E}\left\{\overline{\mathbf{x}}_{e}^{\mathrm{T}}(k) \boldsymbol{\Lambda}_{2} \overline{\mathbf{x}}_{e}(k)\right\}-\sum_{i=k-\tau_{M}}^{k-\tau_{m}-1} \overline{\mathbf{x}}_{e}^{\mathrm{T}}(i) \boldsymbol{\Lambda}_{2} \overline{\mathbf{x}}_{e}(i) .
\end{aligned}
$$

In addition,

$$
\begin{aligned}
& 2 \boldsymbol{\eta}^{\mathrm{T}}(k) \boldsymbol{\Gamma}\left[\overline{\mathbf{x}}(k)-\overline{\mathbf{x}}\left(k-\tau_{M}\right)-\sum_{i=k-\tau_{M}}^{k-1} \overline{\mathbf{x}}_{e}(i)\right]=\mathbf{0}, \\
& 2 \boldsymbol{\eta}^{\mathrm{T}}(k) \boldsymbol{\Psi}\left[\overline{\mathbf{x}}\left(k-\tau_{m}\right)-\overline{\mathbf{x}}\left(k-\tau_{M}\right)-\sum_{i=k-\tau_{M}}^{k-\tau_{m}-1} \overline{\mathbf{x}}_{e}(i)\right]=\mathbf{0},
\end{aligned}
$$

where $\boldsymbol{\eta}^{\mathrm{T}}(k)=\left[\begin{array}{llll}\overline{\mathbf{x}}^{\mathrm{T}}(k) & \overline{\mathbf{x}}^{\mathrm{T}}\left(k-\tau_{m}\right) & \overline{\mathbf{x}}^{\mathrm{T}}(k-\tau(k)) & \overline{\mathbf{x}}^{\mathrm{T}}\left(k-\tau_{M}\right)\end{array}\right]$,

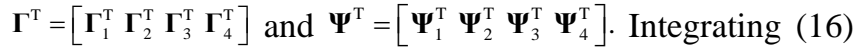
and (17), we get

$$
\begin{aligned}
& \mathrm{E}\{V(\overline{\mathbf{x}}(k+1)) \mid \mathbf{X}(k)\}-V(\overline{\mathbf{x}}(k))+\mathrm{E}\left\{\mathbf{e}^{\mathrm{T}}(k) \mathbf{e}(k)\right\} \\
& \quad-\gamma^{2} \mathrm{E}\left\{\overline{\boldsymbol{\omega}}^{\mathrm{T}}(k) \overline{\boldsymbol{\omega}}(k)\right\} \leq \overline{\boldsymbol{\eta}}^{\mathrm{T}}(k)\left(\boldsymbol{\Sigma}_{\mathbf{\Omega}}^{l}+\boldsymbol{\Sigma}_{M 2}^{\mathrm{T}} \boldsymbol{\Sigma}_{M 3}^{-1} \boldsymbol{\Sigma}_{M 2}\right) \overline{\boldsymbol{\eta}}(k),
\end{aligned}
$$

where $\overline{\boldsymbol{\eta}}^{\mathrm{T}}(k)=\left[\boldsymbol{\eta}^{\mathrm{T}}(k) \overline{\boldsymbol{\omega}}^{\mathrm{T}}(k)\right]$. According to the conditions in (12) and Schur complement, we can make sure that

$$
\boldsymbol{\Sigma}_{\mathbf{\Omega}}^{l}+\boldsymbol{\Sigma}_{M 2}^{\mathrm{T}} \boldsymbol{\Sigma}_{M 3}^{-1} \boldsymbol{\Sigma}_{M 2}<\mathbf{0}
$$

which also implies that

$$
\begin{aligned}
& \mathrm{E}\{V(\overline{\mathbf{x}}(k+1)) \mid \mathbf{X}(k)\}-V(\overline{\mathbf{x}}(k)) \\
& \quad+\mathrm{E}\left\{\mathbf{e}^{\mathrm{T}}(k) \mathbf{e}(k)\right\}-\gamma^{2} \mathrm{E}\left\{\overline{\boldsymbol{\omega}}^{\mathrm{T}}(k) \overline{\boldsymbol{\omega}}(k)\right\}<0
\end{aligned}
$$

Under zero initial conditions, summing (20) from 0 to $\infty$ with respect to $k$, we can find that

$$
\begin{aligned}
\sum_{k=0}^{\infty} E\left\{\mathbf{e}^{\mathrm{T}}(k) \mathbf{e}(k)\right\} \\
\quad<\sum_{k=0}^{\infty} \gamma^{2} \mathrm{E}\left\{\overline{\boldsymbol{\omega}}^{\mathrm{T}}(k) \overline{\boldsymbol{\omega}}(k)\right\}-E\{V(\overline{\mathbf{x}}(\infty)) \mid \mathbf{X}(k)\} .
\end{aligned}
$$

Besides, when $\overline{\boldsymbol{\omega}}(k)=\mathbf{0}$, inequality (20) also implies

$$
\mathrm{E}\{V(\overline{\mathbf{x}}(k+1)) \mid \mathbf{X}(k)\}-V(\overline{\mathbf{x}}(k))<0
$$

Therefore, we can conclude that the augmented estimation dynamics in (8) is stochastically stable and satisfies the $H_{\infty}$ performance in (10) under zero initial conditions. And the proof is completed.

\section{B. Synthesis Conditions for a Design Problem}

In this subsection, a set of LMI-based synthesis conditions of the estimators and controllers design to deal with faults induced by faulty actuators are derived in Theorem 2 .

Theorem 2. If there exist feasible matrices $\mathbf{P}, \mathbf{Q}, \hat{\mathbf{C}}, \mathbf{G}_{a}, \mathbf{G}_{b}, \mathbf{G}_{f}, \mathbf{G}_{k}, \boldsymbol{\Gamma}_{i}, \boldsymbol{\Psi}_{i}(i=1,2,3,4), \mathbf{G}_{j}(j=1,2,3), \boldsymbol{\Phi}_{t}, \boldsymbol{\Lambda}_{t}$ $(t=1,2)$, with nonsingular matrix $\mathbf{G}_{j}$; positive definite matrices $\mathbf{P}, \mathbf{Q}, \Phi_{t}, \boldsymbol{\Lambda}_{t}$, as well as diagonal matrices $\mathbf{P}, \boldsymbol{\Lambda}_{l}, \mathbf{G}_{1}, \mathbf{G}_{3}, \mathbf{\Phi}_{t}$, and block-diagonal matrices $\mathbf{G}_{a}, \mathbf{G}_{b}$, such that the following LMIs in (23) are satisfied for all $l \in \Upsilon$, then the system $\Sigma_{F E C}$ in (8) is stochastically stable and, under zero initial conditions, satisfied an $H_{\infty}$ performance index defined in (10).

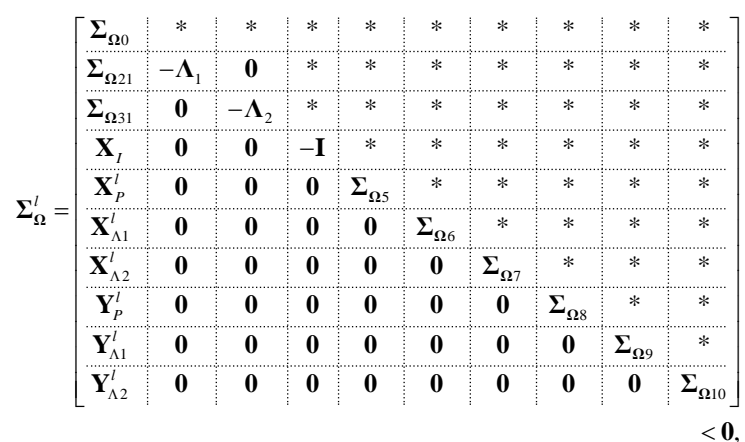


where

$$
\begin{aligned}
& \boldsymbol{\Sigma}_{\boldsymbol{\Omega} 0}=\left[\begin{array}{ccccc}
\left(\tau_{d}+1\right) \mathbf{Q}-\mathbf{P} & & & & \\
+\boldsymbol{\Phi}_{1}+\boldsymbol{\Phi}_{2} & * & * & * & * \\
+\operatorname{sym}\left(\boldsymbol{\Gamma}_{1}\right) & & & & \\
\boldsymbol{\Gamma}_{2}+\boldsymbol{\Psi}_{1}^{\mathrm{T}} & -\boldsymbol{\Phi}_{2}+ & * & * & * \\
\boldsymbol{\Gamma}_{3} & \boldsymbol{s y m}_{3}\left(\boldsymbol{\Psi}_{2}\right) & -\mathbf{Q} & * & * \\
\boldsymbol{\Gamma}_{4}-\boldsymbol{\Gamma}_{1}^{\mathrm{T}} & \boldsymbol{\Psi}_{4}-\boldsymbol{\Gamma}_{2}^{\mathrm{T}} & -\boldsymbol{\Gamma}_{3}^{\mathrm{T}}-\boldsymbol{\Psi}_{3}^{\mathrm{T}} & -\operatorname{sym}\left(\boldsymbol{\Gamma}_{4}\right) & * \\
-\boldsymbol{\Psi}_{1}^{\mathrm{T}} & -\boldsymbol{\Psi}_{2}^{\mathrm{T}} & & -\operatorname{sym}\left(\boldsymbol{\Psi}_{4}\right) & \\
\mathbf{0} & \mathbf{0} & \mathbf{0} & \mathbf{0} & -\gamma^{2} \mathbf{I}
\end{array}\right], \\
& \boldsymbol{\Sigma}_{\mathbf{\Omega} 21}=\sqrt{\tau_{M}}\left[\begin{array}{ll}
\boldsymbol{\Gamma}^{\mathrm{T}} & \mathbf{0}
\end{array}\right] ; \quad \boldsymbol{\Sigma}_{\mathbf{\Omega} 31}=\sqrt{\tau_{d}}\left[\begin{array}{ll}
\boldsymbol{\Psi}^{\mathrm{T}} & \mathbf{0}
\end{array}\right] ; \quad \tau_{d}=\tau_{M}-\tau_{m},
\end{aligned}
$$

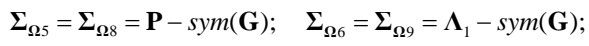

$$
\begin{aligned}
& \Sigma_{\mathbf{\Omega} 7}=\boldsymbol{\Sigma}_{\Omega 10}=\boldsymbol{\Lambda}_{2}-\operatorname{sym}(\mathbf{G}) ; \\
& \mathbf{X}_{I}=\left[\begin{array}{llllll}
\overline{\mathbf{C}}_{1}+\hat{\mathbf{C}} \overline{\mathbf{C}}_{2} & \mathbf{0} & \mathbf{0} & \mathbf{0} & \mathbf{0}
\end{array}\right] \text {; } \\
& \mathbf{X}_{P}^{\prime}=\left[\begin{array}{llllll}
\mathbf{G} \overline{\mathbf{A}}_{1}^{l}+\mathbf{G}_{\mathrm{Am}} \overline{\mathbf{A}}_{2} & \mathbf{0} & \underline{\mathbf{\alpha}} \mathbf{G}_{B m} \overline{\mathbf{B}}_{1}^{l} \quad \mathbf{0} \quad \mathbf{G} \overline{\mathbf{H}}
\end{array}\right] ; \\
& \mathbf{X}_{\Lambda 1}^{l}=\left[\begin{array}{lllllll}
\sqrt{\tau_{M}}\left(\mathbf{G} \overline{\mathbf{A}}_{1}^{l}+\mathbf{G}_{A m} \overline{\mathbf{A}}_{2}-\mathbf{G}\right) & \mathbf{0} & \sqrt{\tau_{M}} \underline{\mathbf{a}} \mathbf{G}_{B m} \overline{\mathbf{B}}_{1}^{l} & \mathbf{0} & \sqrt{\tau_{M}} \mathbf{G} \overline{\mathbf{H}}
\end{array}\right] ; \\
& \mathbf{X}_{\Lambda 2}^{\prime}=\left[\begin{array}{lllllll}
\sqrt{\tau_{d}}\left(\mathbf{G}^{\prime} \overline{\mathbf{A}}_{1}^{l}+\mathbf{G}_{A m} \overline{\mathbf{A}}_{2}-\mathbf{G}\right) & \mathbf{0} & \sqrt{\tau_{d}} \underline{\mathbf{G}_{B m}} \overline{\mathbf{B}}_{1}^{l} & \mathbf{0} & \sqrt{\tau_{d}} \mathbf{G} \overline{\mathbf{H}}
\end{array} ;\right. \\
& \mathbf{Y}_{P}^{\prime}=\left[\begin{array}{llllll}
\mathbf{0} & \mathbf{0} & \sqrt{\boldsymbol{\alpha}_{M}} \mathbf{G}_{B m} \overline{\mathbf{B}}_{1}^{l} & \mathbf{0} & \mathbf{0}
\end{array}\right] ;
\end{aligned}
$$

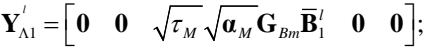

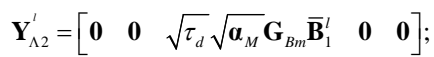

$$
\begin{aligned}
& \boldsymbol{\Gamma}^{\mathrm{T}}=\left[\begin{array}{llll}
\boldsymbol{\Gamma}_{1}^{\mathrm{T}} & \boldsymbol{\Gamma}_{2}^{\mathrm{T}} & \boldsymbol{\Gamma}_{3}^{\mathrm{T}} & \boldsymbol{\Gamma}_{4}^{\mathrm{T}}
\end{array}\right], \boldsymbol{\Psi}^{\mathrm{T}}=\left[\begin{array}{llll}
\boldsymbol{\Psi}_{1}^{\mathrm{T}} & \boldsymbol{\Psi}_{2}^{\mathrm{T}} & \boldsymbol{\Psi}_{3}^{\mathrm{T}} & \boldsymbol{\Psi}_{4}^{\mathrm{T}}
\end{array}\right],
\end{aligned}
$$

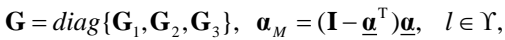

$$
\begin{aligned}
& \overline{\mathbf{A}}_{1}^{l}=\operatorname{diag}\left\{\mathbf{A}_{p}^{l}, \mathbf{A}_{f}, \mathbf{0}\right\}, \overline{\mathbf{A}}_{2}=\left[\begin{array}{lll}
\mathbf{0} & \mathbf{0} & \mathbf{I}
\end{array}\right], \overline{\mathbf{B}}_{1}^{l}=\left[\begin{array}{lll}
\mathbf{C}^{l} & \mathbf{0} & \mathbf{0}
\end{array}\right], \\
& \overline{\mathbf{C}}_{1}=\left[\begin{array}{lll}
\mathbf{0} & \mathbf{C}_{f} & \mathbf{0}
\end{array}\right], \quad \overline{\mathbf{C}}_{2}=\left[\begin{array}{lll}
\mathbf{0} & \mathbf{0} & -\mathbf{I}
\end{array}\right], \\
& \mathbf{G}_{A m}^{\mathrm{T}}=\left[\begin{array}{lll}
\mathbf{G}_{f}^{\mathrm{T}} & \mathbf{0} & \mathbf{G}_{a}^{\mathrm{T}}
\end{array}\right], \mathbf{G}_{B m}^{\mathrm{T}}=\left[\begin{array}{lll}
\mathbf{G}_{k}^{\mathrm{T}} & \mathbf{0} & \mathbf{G}_{b}^{\mathrm{T}}
\end{array}\right] .
\end{aligned}
$$

If in this case, the estimator gains and controller gains can be found by

$$
\begin{aligned}
& \hat{\mathbf{A}}=\mathbf{G}_{3}^{-1} \mathbf{G}_{a}, \quad \hat{\mathbf{B}}=\mathbf{G}_{3}^{-1} \mathbf{G}_{b}, \quad \hat{\mathbf{C}}, \\
& \mathbf{K}=\left(\mathbf{B}^{\mathrm{T}} \mathbf{B}\right)^{-1} \mathbf{B}^{\mathrm{T}} \mathbf{G}_{1}^{-1} \mathbf{G}_{k}, \\
& \mathbf{K}_{f}=\left(\mathbf{B}^{\mathrm{T}} \mathbf{B}\right)^{-1} \mathbf{B}^{\mathrm{T}} \mathbf{G}_{1}^{-1} \mathbf{G}_{f} \hat{\mathbf{C}}^{\mathrm{T}}\left(\hat{\mathbf{C}} \hat{\mathbf{C}}^{\mathrm{T}}\right)^{-1} .
\end{aligned}
$$

Proof. Apply the stability conditions in (12) and Lemma 1, as well as set

$$
\mathbf{G}_{a}=\mathbf{G}_{3} \hat{\mathbf{A}}, \quad \mathbf{G}_{b}=\mathbf{G}_{3} \hat{\mathbf{B}}, \quad \mathbf{G}_{k}=\mathbf{G}_{1} \mathbf{B K}, \quad \mathbf{G}_{f}=\mathbf{G}_{1} \mathbf{B} \mathbf{K}_{f} \hat{\mathbf{C}}
$$

we can easily derive the synthesis conditions in (23) for the estimators and controllers design.

\section{NUMERICAL EXAMPLE}

As shown in Fig. 1, consider the system (1) with two subsystems, whose matrices defined in (2) are as follows:

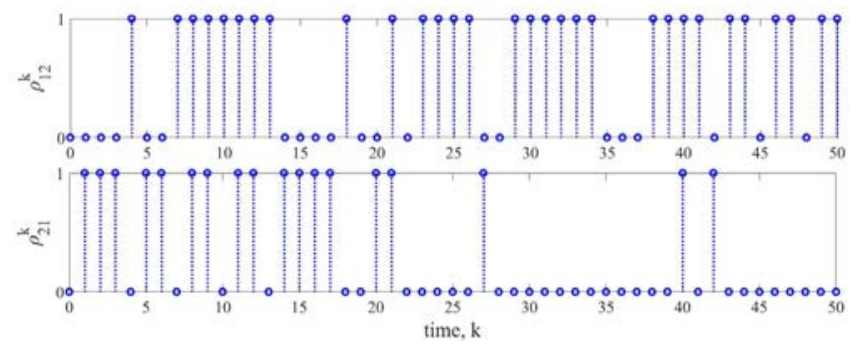

Fig. 2. Time-varying coupling parameters.
$\mathbf{A}^{\left(\rho_{k}\right)}=\left[\begin{array}{cc}\mathbf{A}_{1} & \rho_{12}^{k} \mathbf{A}_{12} \\ \rho_{21}^{k} \mathbf{A}_{21} & \mathbf{A}_{2}\end{array}\right], \mathbf{C}^{\left(\rho_{k}\right)}=\left[\begin{array}{cc}\mathbf{C}_{1} & \rho_{12}^{k} \mathbf{C}_{12} \\ \rho_{21}^{k} \mathbf{C}_{21} & \mathbf{C}_{2}\end{array}\right], \mathbf{F}^{\left(\rho_{k}\right)}=\left[\begin{array}{cc}\mathbf{F}_{1} & \rho_{12}^{k} \mathbf{F}_{12} \\ \rho_{21}^{k} \mathbf{F}_{21} & \mathbf{F}_{2}\end{array}\right]$, $\mathbf{B}=\operatorname{diag}\left\{\mathbf{B}_{1}, \mathbf{B}_{2}\right\}, \quad \mathbf{D}=\operatorname{diag}\left\{\mathbf{D}_{1}, \mathbf{D}_{2}\right\}$,

$$
\begin{aligned}
& {\left[\begin{array}{cccc}
\mathbf{A}_{1} & \mathbf{A}_{12} & \mathbf{C}_{1} & \mathbf{C}_{12} \\
\mathbf{A}_{21} & \mathbf{A}_{2} & \mathbf{C}_{21} & \mathbf{C}_{2}
\end{array}\right]=} \\
& {\left[\begin{array}{cccccccc}
0.12 & -0.096 & 0.125 & 0 & 0.45 & -0.9 & 1 & 0 \\
0 & 0.24 & 0 & -0.25 & 0.45 & 0.45 & 0 & -1 \\
0.025 & 0 & 0.14 & 0.1 & 0.1 & 0 & 0.5 & 0.9 \\
0 & -0.0125 & 0 & 0.26 & 0 & -0.1 & -1 & 0.5
\end{array}\right],}
\end{aligned}
$$

$\left[\begin{array}{llllllll}\mathbf{B}_{1} & \mathbf{B}_{2} & \mathbf{F}_{1} & \mathbf{F}_{2} & \mathbf{F}_{12} & \mathbf{F}_{21} & \mathbf{D}_{1} & \mathbf{D}_{2}\end{array}\right]=$

$\left[\begin{array}{cccccccccc}0 & -1 & 1 & 1 & 1.2 & 1.2 & 0.1 & 0 & 1 & 1 \\ 2 & 1 & 2 & 0 & 0.4 & 0.4 & 0 & -0.01 & 1 & 1\end{array}\right]$.

Fig. 2 shows the corresponding time-varying coupling parameters $\rho_{i j(i, j=\{1,2\}, i \neq j)}^{k}$, which indicates the types of configuration between these two subsystems in the NCS. The fault dynamics (3) induced by faulty actuators is supposed to be with

$$
\begin{aligned}
& \mathbf{A}_{f}=\operatorname{diag}\left\{\mathbf{A}_{f 1}, \mathbf{A}_{f 2}\right\}, \mathbf{B}_{f}=\operatorname{diag}\left\{\mathbf{B}_{f 1}, \mathbf{B}_{f 2}\right\}, \mathbf{C}_{f}=\operatorname{diag}\left\{\mathbf{C}_{f 1}, \mathbf{C}_{f 2}\right\}, \\
& {\left[\begin{array}{ll}
\mathbf{A}_{f 1} & \mathbf{A}_{f 2} \\
\mathbf{B}_{f 1}^{\mathrm{T}} & \mathbf{B}_{f 2}^{\mathrm{T}} \\
\mathbf{C}_{f 1} & \mathbf{C}_{f 2}
\end{array}\right]=\left[\begin{array}{cccccc}
0.26 & 0 & 0.052 & 0.26 & 0 & 0.052 \\
0 & 0.78 & -0.078 & 0 & 0.78 & -0.078 \\
0.26 & 0.13 & 0.26 & 0.26 & 0.13 & 0.26 \\
-0.25 & 0.165 & 0.07 & -0.25 & 0.165 & 0.07 \\
0.4 & 1.2 & 0.8 & 0.4 & 0.32 & 0.2
\end{array}\right],}
\end{aligned}
$$

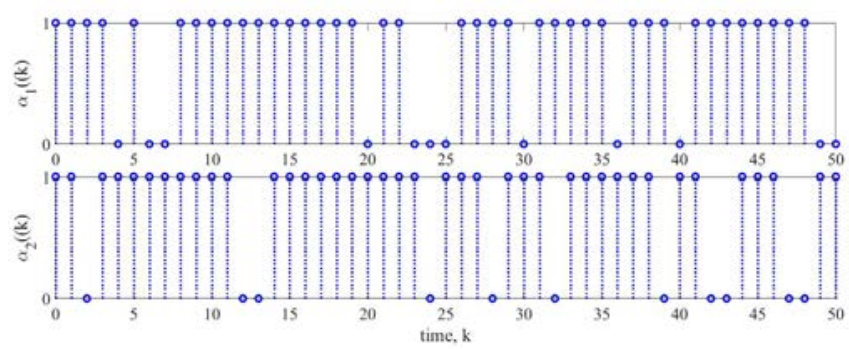

Fig. 3. Flags of data package received or lost.

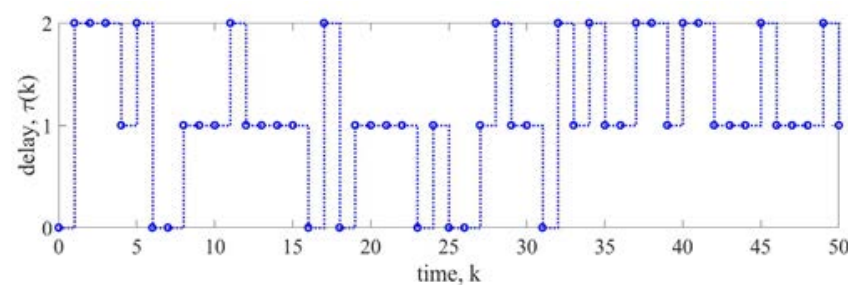

Fig. 4. Networked induced time-delay.

For the NCS, $\alpha_{1}=\alpha_{2}=0.8$ and the networked induced time-varying delay $0 \leq\left\{\tau(k),\left(\tau_{1}(k), \tau_{2}(k)\right)\right\} \leq 2$. Fig. 3 and Fig. 4 show the corresponding practical events related to the packet received/lost and time-varying delay, respectively. Suppose the desired $H_{\infty}$ performance index is $\gamma=1.732$. Using the synthesis conditions described in Theorem 2, the design problem which is a feasibility problem under the linear matrix inequality framework, has a set of feasible solutions and the gains of the estimators and controllers for faults can be found as 
$\hat{\mathbf{A}}=\operatorname{diag}\left\{\hat{\mathbf{A}}_{1}, \hat{\mathbf{A}}_{2}\right\}, \hat{\mathbf{B}}=\operatorname{diag}\left\{\hat{\mathbf{B}}_{1}, \hat{\mathbf{B}}_{2}\right\}, \hat{\mathbf{C}}=\operatorname{diag}\left\{\hat{\mathbf{C}}_{1}, \hat{\mathbf{C}}_{2}\right\}$,

$$
\begin{aligned}
& {\left[\begin{array}{lll}
\hat{\mathbf{A}}_{1} & \hat{\mathbf{B}}_{1} & \hat{\mathbf{C}}_{1}^{\mathrm{T}} \\
\hat{\mathbf{A}}_{2} & \hat{\mathbf{B}}_{2} & \hat{\mathbf{C}}_{2}^{\mathrm{T}}
\end{array}\right]=} \\
& {\left[\begin{array}{rrrrr}
0.7245 & 0.0006 & -7.32 \times 10^{-5} & 2.38 \times 10^{-5} & -2.3698 \\
-0.0012 & 0.7260 & 1.92 \times 10^{-5} & -3.66 \times 10^{-5} & -1.0922 \\
0.7256 & 0.0007 & -1.267 \times 10^{-4} & -4.78 \times 10^{-5} & -0.4117 \\
0.0006 & 0.7265 & -4.49 \times 10^{-5} & -1.045 \times 10^{-4} & -0.1034
\end{array}\right]} \\
& \mathbf{K}=\operatorname{diag}\left\{\mathbf{K}_{1}, \mathbf{K}_{2}\right\}, \mathbf{K}_{f}=\operatorname{diag}\left\{\mathbf{K}_{f 1}, \mathbf{K}_{f 2}\right\}, \\
& {\left[\begin{array}{cc}
\mathbf{K}_{1} & \mathbf{K}_{2} \\
\mathbf{K}_{f 1}^{\mathrm{T}} & \mathbf{K}_{f 2}^{\mathrm{T}}
\end{array}\right]=\left[\begin{array}{rrrr}
-0.0034 & 0.0010 & -0.0007 & -0.0010 \\
0.0065 & -0.0031 & -0.0038 & -0.0000 \\
2.0297 & -3.0649 & 1.6970 & 7.5577
\end{array}\right] .}
\end{aligned}
$$

Under the consideration with the practical transmission and delay shown in Fig. 3 and Fig. 4, and $\overline{\mathbf{f}}(k)=\left[\begin{array}{ll}0.1 & 0.1\end{array}\right]^{\mathrm{T}} \delta(k), \boldsymbol{\omega}(k)=\left[\begin{array}{ll}1 & 1\end{array}\right]^{\mathrm{T}} 0.01 e^{-0.1 k} \sin (k-1)$, the estimation errors for faults of the controlled NCS with $\rho_{i j(i, j=\{1,2\}, i \neq j)}^{k}$ shown in Fig. 2, are presented in Fig. 5, while the states responses of the controlled NCS are shown in Fig. 6. Fig. 5 and Fig. 6 indicate that the feasibility of the proposed method.

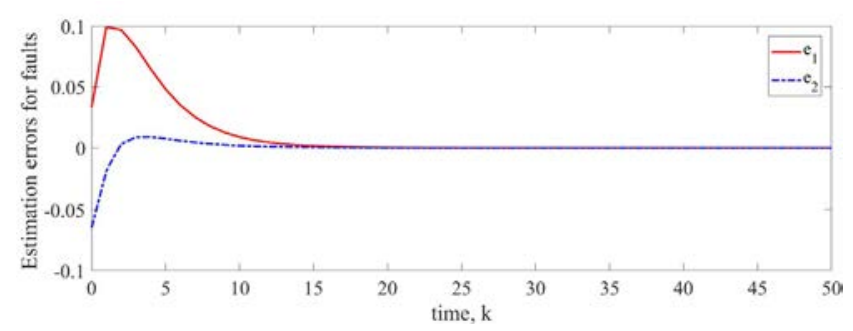

Fig. 5. Estimation errors for faults.

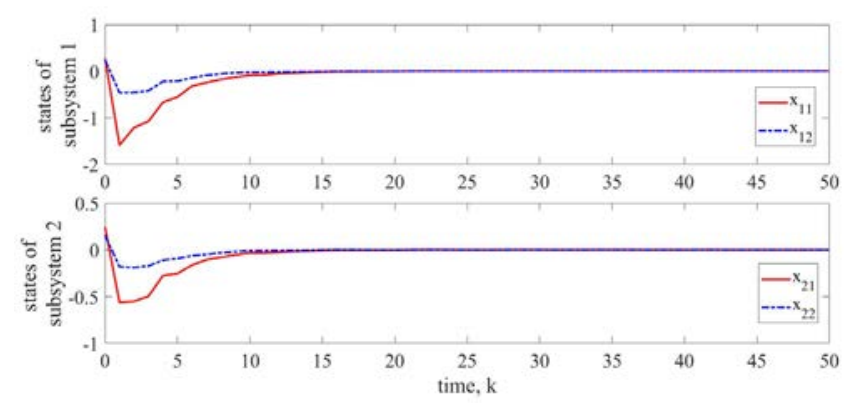

Fig. 6. States responses of these two subsystems.

\section{CONCLUSION}

In this paper, a faults estimation and control problem for an integrated NCS whose configuration between subsystems is with variable structures are investigated. The contribution of this paper at least includes two aspects. First one is that the formulation allows taking into account a distributed NCS, or a decentralized one, or a time-varying kind switching between a distributed and decentralized NCS because of introducing the coupling parameters related to the variable structures. And the second one is that a set of LMI-based delay-dependent conditions for stability analysis problems and for synthesis problems related to the estimators and controllers are derived for such NCS.

\section{REFERENCES}

[1] V. Radisavljevic-Gajic, S. Park, and D. Chasaki, "Vulnerabilities of control systems in Internet of Things applications," IEEE Internet of Things Journal, vol. 5, no. 2, pp. 1023-1032, 2018.

[2] M. S. Mahmoud and M. M. Hamdan, "Fundamental issues in networked control systems,” IEEE/CAA Journal of Automatica Sinica, vol. 5, no. 5, pp. 902-922, 2018.

[3] X. Zhang, Q. Han, and X. Yu, "Survey on recent advances in networked control systems,” IEEE Transactions on Industrial Informatics, vol. 12, no. 5, pp. 1740-1752, 2016.

[4] X. Ge, F. Yang, and Q.-L. Han, "Distributed networked control systems: A brief overview," Information Sciences, vol. 380, pp. 117-131, 2017.

[5] X. Zhang, Q. Han, and B. Zhang, “An overview and deep investigation on sampled-data-based event-triggered control and filtering for networked systems," IEEE Transactions on Industrial Informatics, vol. 13, no. 1, pp. 4-16, 2017.

[6] L. Su and G. Chesi, "Robust stability analysis and synthesis for uncertain discrete-time networked control systems over fading channels,” IEEE Transactions on Automatic Control, vol. 62, no. 4, pp. 1966-1971, 2017.

[7] D. Zhang, S. K. Nguang, and L. Yu, "Distributed control of large-scale networked control systems with communication constraints and topology switching," IEEE Transactions on Systems, Man, and Cybernetics: Systems, vol. 47, no. 7, pp. 1746-1757, 2017.

[8] I. Chen, A. P. Speer, and M. Eltoweissy, “Adaptive fault-tolerant qos control algorithms for maximizing system lifetime of query-based wireless sensor networks," IEEE Transactions on Dependable and Secure Computing, vol. 8, no. 2, pp. 161-176, 2011.

[9] L. Zhu, F. R. Yu, T. Tang, and B. Ning, “An integrated train-ground communication system using wireless network virtualization: security and quality of service provisioning," IEEE Transactions on Vehicular Technology, vol. 65, no. 12, pp. 9607-9616, 2016.

[10] H. Mo, W. Wang, M. Xie, and J. Xiong, "Modeling and analysis of the reliability of digital networked control systems considering networked degradations," IEEE Transactions on Automation Science and Engineering, vol. 14, no. 3, pp. 1491-1503, 2017.

[11] K. Schenk, B. Gülbitti, and J. Lunze, "Cooperative fault-tolerant control of networked control systems," IFAC-PapersOnLine, vol. 51, no. 24, pp. 570-577, 2018.

[12] S. X. Ding, “A survey of fault-tolerant networked control system design,” IFAC Proceedings Volumes, vol. 45, no. 20, pp. 874-885, 2012.

[13] C.-M. Lee, Y.-C. Huang, and C.-C. Kung, "Faults estimation and decentralized control for an integrated networked control system with faulty actuators," in Proc. the 6th International Conference on Industrial Engineering and Applications, 2019.

[14] M. C. Oliveira, J. Bernussou, and J. C. Geromel, “A new discrete-time robust stability condition,” Systems \& Control Letters, vol. 37, no. 4, pp. 261-265, 1999.

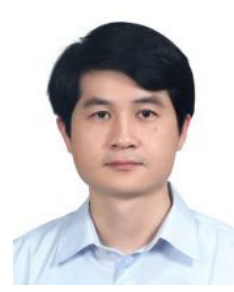

Ching-Min Lee received his Ph.D. degree in electrical engineering from National Taiwan University in Taiwan. He is an assistant professor of Electrical Engineering Department at I-Shou University, Taiwan. His current research interests include the areas of robust control, networked control systems, and signal processing. 